SMAD, Rev. Eletrônica Saúde Mental Álcool Drog.

2020 jan.-fev.; 16(1):1-8

DOI: 10.11606/issn. 1806-6976.smad.2020.156687

www.revistas.usp.br/smad/

Artigo Original

\title{
Perfil epidemiológico dos usuários de um Centro de Atenção Psicossocial
}

Camila Gomes Barbosa ${ }^{1}$

(D) https://orcid.org/0000-0003-2634-9721

Paulo Roberto Marinho Meira ${ }^{1}$

(D) https://orcid.org/0000-0002-6884-2277

Joilda Silva Nery²

(D) https://orcid.org/0000-0002-1576-6418

Bruno Bezerra Gondim ${ }^{1}$

(D) https://orcid.org/0000-0001-9825-9771
${ }^{1}$ Universidade Federal do Vale do São Francisco, Departamento de Medicina, Paulo Afonso, BA, Brasil.

2 Universidade Federal da Bahia, Departamento de Saúde Coletiva, Salvador, BA, Brasil.
Objetivo: analisar as características clínicas e sociodemográficas dos usuários do Centro de Atenção Psicossocial II (CAPS II) do município de Paulo Afonso - Bahia. Método: trata-se de um estudo quantitativo e transversal, realizado a partir do levantamento de dados dos pacientes frequentadores do CAPS II de Paulo Afonso, no ano de 2018. Aspectos clínicos e sociodemográficos foram obtidos a partir dos prontuários médicos dos usuários ativos do serviço cujos anos de cadastramento variaram de 2006 a 2018. Resultados: prevaleceram o sexo feminino, o estado civil solteiro, o Ensino Fundamental incompleto e o analfabetismo, a faixa etária entre 40 e 49 anos, os indivíduos ocupados, a assistência não intensiva e a admissão por encaminhamento. O diagnóstico mais frequente foi do grupo dos transtornos esquizofrênicos, esquizotípicos e delirantes. Os diagnósticos associaram-se significativamente à variável "sexo", sendo o gênero masculino com maior prevalência de transtornos nos grupos dos transtornos mentais orgânicos, esquizofrenia, transtornos esquizotípicos e delirantes e retardo mental. Já o feminino obteve maior proporção nos transtornos do humor e transtornos neuróticos relacionados com o estresse e somatoformes. Conclusão: os dados obtidos neste estudo possuem relevância para contribuir com o direcionamento de estratégias de aprimoramento e organização do Centro de Atenção Psicossocial de Paulo Afonso.

Descritores: Transtornos Mentais; Perfil Epidemiológico; Centros de Atenção Psicossocial.

\section{Como citar este artigo}

Barbosa CG, Meira PRM, Nery JS, Gondim BB. Epidemiological profile of the users of a Psychosocial Care Center. SMAD, Rev Eletrônica Saúde Mental Álcool Drog. 2020;16(1):1-8. doi: https://dx.doi.org/10.11606/issn.1806-6976.smad.2020.156687 


\section{Epidemiological profile of the users of a Psychosocial Care Center}

Objective: to analyze the clinical and sociodemographic characteristics of users of the Psychosocial Care Center II (CAPS II) in the city of Paulo Afonso - Bahia. Method: this is a quantitative and cross-sectional study, conducted from the survey of data from patients attending CAPS II of Paulo Afonso, in 2018. Clinical and sociodemographic aspects were obtained from the medical records of the active users of the service whose years of registration ranged from 2006 to 2018. Results: prevailed female gender, single marital status, incomplete elementary school and illiteracy, age between 40 and 49 years, employed persons, non-intensive care and admission by referral. The most frequent diagnosis was in the group of schizophrenic, schizotypic and delusional disorders. The diagnoses were significantly associated with the variable "gender", being the male gender with higher prevalence of disorders in the groups of organic mental disorders, schizophrenia, schizotypic and delusional disorders and mental retardation. The female had a higher proportion in mood disorders and neurotic disorders related to stress and somatoforms. Conclusion: the data obtained in this study are relevant to contribute to the direction of improvement strategies and organization of the Paulo Afonso Psychosocial Care Center.

Descriptors: Mental Disorders; Epidemiological Profile, Psychosocial Care Center.

\section{Perfil epidemiológico de los usuarios de un Centro de Atención Psicosocial}

Objetivo: analizar las características clínicas y sociodemográficas de los usuarios del Centro de Atención Psicosocial II (CAPS II) del municipio de Paulo Afonso - Bahia. Método: se trata de un estudio cuantitativo y transversal, realizado a partir del levantamiento de datos de los pacientes frecuentadores del CAPS II de Paulo Afonso en el año 2018. Aspectos clínicos y sociodemográficos fueron obtenidos a partir de los prontuarios médicos de los usuarios activos del servicio, de los cuales, en los años de registro, varían de 2006 a 2018. Resultados: prevalece el sexo femenino, el estado civil soltero, la enseñanza fundamental incompleta y analfabetismo, el grupo de edad entre 40 y 49 años, los individuos ocupados, la asistencia no intensiva y la admisión por enrutamiento. El diagnóstico más frecuente fue del grupo de los trastornos esquizofrénicos, esquizotípicos y delirantes. Los diagnósticos se asociaron significativamente a la variable "sexo", siendo género masculino con mayor prevalencia de trastornos en los grupos de los trastornos mentales orgánicos; esquizofrenia, trastornos esquizotípicos y delirantes; y retraso mental. Ya lo femenino, obtuvo mayor proporción en los trastornos del humor; y trastornos neuróticos, relacionados con el estrés y somatoformes. Conclusión: los datos obtenidos en este estudio poseen relevancia para contribuir con el direccionamiento de estrategias de perfeccionamiento y organización del Centro de Atención Psicosocial de Paulo Afonso.

Descriptores: Trastornos Mentales; Perfil Epidemiológico; Centros de Atención Psicosocial. 


\section{Introdução}

Os transtornos mentais são síndromes que interferem em processos biológicos e psicológicos fundamentais para a regulação emocional, cognitiva e comportamental dos indivíduos. Assim, relacionam-se ao sofrimento e ao comprometimento de atividades da vida pessoal e laboral dos acometidos ${ }^{(1)}$. Essas desordens merecem atenção visto que atingem milhões de pessoas no mundo e apresentam altos níveis de discriminação, mortes precoces e abuso físico e sexual(2). Estima-se que, de quatro indivíduos, um irá ser acometido por transtornos mentais em algum momento da vida( ${ }^{(3)}$. Desse modo, é importante que a rede de saúde mental esteja preparada para acolher e tratar seus pacientes de maneira adequada.

No entanto, por muito tempo, a assistência restringiu-se ao modelo manicomial, centrado em internações de longa duração em hospitais psiquiátricos, onde os maus-tratos eram recorrentes. A fim de mudar esse cenário do Brasil, instaurou-se a reforma psiquiátrica brasileira, que consiste em um processo histórico o qual foi pautado na crítica à assistência supracitada. Esse movimento, iniciado a partir de 1980, possuía particularidades de acordo com a região do país e foi influenciado por várias correntes, pelo contexto histórico da época e pelo êxito de substituição do modelo supracitado em outros países. Além disso, envolveu mobilizações nas quais os trabalhadores da saúde, pessoas em sofrimento psíquico e seus familiares almejavam um cuidado mais humanizado(4-5).

Esses movimentos repercutiram positivamente, culminando, ao longo dos anos, em várias medidas como a sanção da Lei Federal no 10.216, a qual foi fundamental para a consolidação da política de saúde mental do governo federal. Essa lei afirma os direitos e a proteção das pessoas com transtornos mentais e redireciona o modelo de assistência em saúde mental para um modelo de atenção comunitário(6) por meio de redução de leitos de hospitais psiquiátricos e estabelecimento de serviços substitutivos à atenção psiquiátrica hospitalocêntrica antes vigente, como os Centros de Atenção Psicossocial (CAPS), os Serviços Residenciais Terapêuticos (SRT) e as próprias Unidades Básicas de Saúde (UBS) ${ }^{(4,6)}$.

O CAPS é um tipo de serviço de saúde de caráter aberto, comunitário e multidisciplinar, que oferece atendimento aos pacientes com TM persistentes e graves e àqueles com problemas relacionados ao uso de álcool e outras drogas em determinada unidade territorial(7). Essas instituições têm como principal objetivo o acolhimento desse público, sua integração aos meios social e familiar e a disponibilização de apoio à busca por autonomia e de atendimento médico e psicológico( ${ }^{(8)}$. Esses centros organizam-se em diferentes tipos de acordo com sua complexidade, o público-alvo e o número de habitantes do local. Desse modo, classificam-se em CAPS I, II, III, Álcool e Drogas (AD), AD III, Infantil (i) ${ }^{(7)}$.

Nesses estabelecimentos, o processo de trabalho dá-se de acordo com a gravidade do quadro dos usuários, sendo priorizados os casos de transtornos de maior gravidade nesse atendimento. Essa assistência pode ser intensiva, semi-intensiva e não intensiva e exige acompanhamento diário, frequente e menos frequente, respectivamente ${ }^{(9)}$.

Com uma população estimada, para o ano de 2018, de 117.014 indivíduos(10), o município de Paulo Afonso/BA possui, conforme o Cadastro Nacional de Estabelecimentos de Saúde(11), uma unidade de CAPS II e outra de CAPS-ad, sendo a instalação de ambas preconizada em locais cujo número de habitantes supere $70 \mathrm{mil}^{(7)}$. O local deste estudo foi CAPS II de Paulo Afonso/BA, o qual foi criado em 2006 e realiza atendimento por meio de uma equipe multidisciplinar.

Objetivou-se, por este trabalho, considerando que não há estudos que abordem a caracterização o CAPS II de Paulo Afonso/BA, que o novo modelo de assistência à saúde mental ainda se encontra em transição e que o conhecimento dos aspectos clínicos e sociodemográficos do público atendido em determinado serviço de saúde é essencial para nortear o planejamento de estratégias para a sua melhoria, analisar as características referentes à idade, sexo, etnia, estado civil, nível de escolaridade, ocupação, cidade de residência, tipo de demanda, modalidade de tratamento e diagnóstico dos usuários do Centro de Atenção Psicossocial II do município de Paulo Afonso - Bahia.

\section{Método}

Foi realizado um estudo de caráter transversal, no Centro de Atenção Psicossocial II do município de Paulo Afonso/BA, no período compreendido entre abril e junho de 2018.

Durante o período do estudo, foram incluídos todos os indivíduos ativos (cadastrados e frequentadores do serviço) de todas as faixas etárias, regimes de atendimento (não intensivo, semi-intensivo ou intensivo) e cidades de residência, sendo excluídos do levantamento apenas os não frequentadores dos CAPS II. A partir desses critérios, analisaram-se 293 prontuários cujos anos de admissão dos pacientes variaram de 2006 a 2018.

Os dados para análise do perfil clínico e sociodemográfico foram extraídos dos prontuários médicos, sendo eles referentes à modalidade de atendimento atual, diagnóstico psiquiátrico mais recente, tipo de demanda, idade, sexo, estado civil, raça/cor, nível de escolaridade e ocupação. As hipóteses diagnósticas foram apresentadas em grupos de acordo com a décima versão da Classificação internacional de Doenças $(C I D)^{(12)}$. Foram calculadas as frequências simples e 
percentuais das variáveis do estudo. Posteriormente, aplicou-se o teste exato de Fisher $^{(13)}$ para a verificação de associação entre o sexo e os diagnósticos clínicos dos usuários. O nível de significância adotado foi inferior a 0,05 e as informações obtidas no estudo foram armazenadas e analisadas por meio dos programas Microsoft Excel 2010 e Stata, versão 12.0.

Este trabalho seguiu a Resolução n. 466/2012 do Conselho Nacional de Saúde e foi aprovado com parecer número 2.547.699 pelo Comitê de Ética e Pesquisa da Universidade Federal do Vale do São Francisco (UNIVASF).

\section{Resultados}

Foram analisados os prontuários dos 293 usuários ativos do CAPS II do município de Paulo Afonso/BA. A amostra estudada apresentou maior proporção de indivíduos na faixa etária entre 40 e 49 anos (37,88\%). Com relação ao sexo, constatou-se que 163 pacientes eram mulheres (55,63\%) e 130, homens (44,37\%). Já no que tange ao estado civil, a maioria dos indivíduos encontrava-se solteira (165/56,31\%) (Tabela 1).

Tabela 1 - Características sociodemográficas dos usuários do CAPS II* de Paulo Afonso, BA, Brasil, 2018

\begin{tabular}{|c|c|c|}
\hline Variável & $\mathbf{N}$ & $\%$ \\
\hline \multicolumn{3}{|l|}{ Sexo } \\
\hline Feminino & 163 & 55,63 \\
\hline Masculino & 130 & 44,37 \\
\hline \multicolumn{3}{|l|}{ Faixas etárias } \\
\hline 15 a 19 anos & 6 & 2,05 \\
\hline 20 a 29 anos & 25 & 8,53 \\
\hline 30 a 39 anos & 74 & 25,26 \\
\hline 40 a 49 anos & 111 & 37,88 \\
\hline 50 a 59 anos & 57 & 19,45 \\
\hline 60 ou mais & 20 & 6,83 \\
\hline \multicolumn{3}{|l|}{ Estado civil } \\
\hline Solteiro & 165 & 56,31 \\
\hline Casado & 68 & 23,21 \\
\hline Divorciado & 12 & 4,10 \\
\hline Viúvo & 8 & 2,73 \\
\hline União estável & 5 & 1,71 \\
\hline Não informado & 35 & 11,95 \\
\hline \multicolumn{3}{|l|}{ Ocupação } \\
\hline Desempregado & 25 & 8,53 \\
\hline Empregado & 104 & 35,49 \\
\hline Estudante & 4 & 1,37 \\
\hline Aposentado & 8 & 2,73 \\
\hline Não informado & 152 & 51,88 \\
\hline \multicolumn{3}{|l|}{ Escolaridade } \\
\hline $\begin{array}{l}\text { Não alfabetizado/ } \\
\text { E. Fundamental incompleto }\end{array}$ & 107 & 36,52 \\
\hline E. Fundamental completo & 12 & 4,10 \\
\hline E. Médio incompleto & 11 & 3,75 \\
\hline E. Médio completo & 27 & 9,22 \\
\hline E. Superior incompleto & 2 & 0,68 \\
\hline E. Superior completo & 2 & 0,68 \\
\hline Outros $^{\dagger}$ & 11 & 3,76 \\
\hline Não informado & 121 & 41.30 \\
\hline
\end{tabular}

*Centro de Atenção Psicossocial II; 'Estudo com limitações; assina o nome; alfabetizado; magistério; frequentador da APAE
Em relação à ocupação, a maior fração dos usuários estava empregada (104/35,49\%). Quanto à escolaridade, $121(41,3 \%)$ não possuíam dados preenchidos a respeito dessa variável. Entre aqueles que tinham informações sobre esse aspecto, a maioria $(107 / 36,52 \%)$ apresentou Ensino Fundamental incompleto e analfabetismo e a minoria, Ensino Médio incompleto e completo em igual proporção (2/0,68\%) (Tabela 1).

No que concerne à cidade de residência, apenas um dos usuários não residia no município de Paulo Afonso/ BA. Já em relação à variável raça/cor, somente nove prontuários continham esse dado.

Dos diagnósticos clínicos, agrupados nas categorias da Classificação Internacional de Doenças (CID-10), os mais prevalentes foram os transtornos do grupo da esquizofrenia, transtornos esquizotípicos e delirantes, com 121 indivíduos $(41,3 \%)$, seguidos dos transtornos do humor [afetivos], presentes em 53 pacientes $(18,09 \%)$, e dos diagnósticos combinados de 50 usuários $(17,06 \%)$ (Tabela 2).

Tabela 2 - Distribuição segundo o diagnóstico clínico dos usuários do CAPS II* de Paulo Afonso, BA, Brasil, 2018

\begin{tabular}{lcc}
\multicolumn{1}{c}{ CID10 $^{\dagger}$} & N & $\%$ \\
\hline $\begin{array}{l}\text { F00-F09: Transtornos mentais orgânicos, } \\
\text { inclusive os sintomáticos }\end{array}$ & 13 & 4,44 \\
F10 a F19: Transtornos mentais e & 2 & 0,68 \\
comportamentais devido ao uso de substância & & \\
psicoativa & & \\
F20 a F29: Esquizofrenia, transtornos & 121 & 41,30 \\
esquizotípicos e transtornos delirantes & & \\
F30 a F39: Transtornos do humor [afetivos] & 53 & 18,09 \\
F40 a F48: Transtornos neuróticos relacionados & 21 & 7,17 \\
com o estresse e somatoformes & & \\
F70-F79: Retardo mental & 29 & 9,90 \\
Mais de um diagnóstico & 50 & 17,06 \\
Não definido & 4 & 1,37 \\
Total & 293 & 100 \\
\hline
\end{tabular}

*Centro de Atenção Psicossocial II; ${ }^{+}$CID10 = Código Internacional de Doenças $10^{\mathrm{a}}$ edição

Observou-se, ainda, que o regime de atendimento predominante correspondeu ao não intensivo (210/71,67\%), seguido do semi-intensivo (60/20,48\%) e intensivo (22/7,51\%). Outro dado encontrado foi a admissão de 165 pacientes (56,31\%) no CAPS II por meio de encaminhamentos e de 128 usuários (43,69\%) por demanda espontânea.

Por meio de associação entre os diagnósticos clínicos e a variável "sexo", constatou-se que os transtornos mentais orgânicos (F00-F09), a esquizofrenia, os transtornos esquizotípicos e delirantes (F20-F29) e o retardo mental (F70-F79) predominaram no sexo masculino, já os transtornos do humor (F30-F39) e transtornos neuróticos, relacionados com o estresse e somatoformes (F40-F48), foram mais prevalentes no feminino, com significância estatística $(p=0,00)$ (Tabela 3$)$. 
Tabela 3 - Associação entre o diagnóstico clínico com o sexo dos usuários do CAPS II* de Paulo Afonso, BA, Brasil, 2018

\begin{tabular}{rccccccccc}
\hline CID10 & $\mathbf{F 0 0 - F 0 9}$ & $\mathbf{F 1 0 - F 1 9}$ & $\mathbf{F 2 0 - F 2 9}$ & $\mathbf{F 3 0 - F 3 9}$ & $\mathbf{F 4 0 - F 4 8}$ & $\mathbf{F 7 0 - F 7 9}$ & $\begin{array}{c}\text { Mais de um } \\
\text { transtorno }\end{array}$ & $\begin{array}{c}\text { Não } \\
\text { definido }\end{array}$ & p-valor $^{\ddagger}$ \\
\cline { 2 - 9 } Variáveis & $\mathbf{n}(\%)$ & $\mathbf{n}(\%)$ & $\mathbf{n}(\%)$ & $\mathbf{n}(\%)$ & $\mathbf{n}(\%)$ & $\mathbf{n}(\%)$ & $\mathbf{n}(\%)$ & $\mathbf{n}(\%)$ \\
\hline Sexo & & & & & & & & & \\
Feminino & $4(30,77)$ & $1(50)$ & $52(42,98)$ & $45(84,91)$ & $19(90,48)$ & $10(34,48)$ & $30(60)$ & $2(50)$ & 0,00 \\
Masculino & $9(69,23)$ & $1(50)$ & $69(57,02)$ & $8(15,09)$ & $2(9,52)$ & $19(65,52)$ & $20(40)$ & $2(50)$ & \\
\hline
\end{tabular}

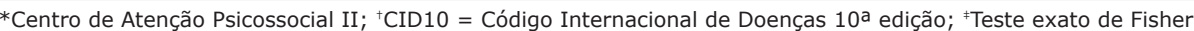

\section{Discussão}

O predomínio da população adulta e economicamente ativa deste estudo assemelha-se ao que foi encontrado em outras pesquisas sobre a epidemiologia dos transtornos mentais(14-18). Dentre os possíveis fatores determinantes para o adoecimento dessa faixa etária encontram-se a habitação precária, o desemprego, o estado civil separado/divorciado/ viúvo e não acesso aos bens de consumo(19). Apesar de a maioria dos prontuários $(51,88 \%)$ não ter apresentado informações sobre ocupação, a maior proporção encontrada $(35,49 \%)$ foi dos pacientes que se encontravam empregados, o que é um ponto positivo, uma vez que trabalhar contribui para a autovalorização do indivíduo, ao reafirmar sua capacidade, que é culturalmente depreciada muitas vezes $^{(20)}$. Além disso, um estudo prospectivo realizado em Vancouver, Canadá, mostrou que a aquisição de um emprego aumentou o bem-estar psicológico de pessoas com transtornos mentais graves ${ }^{(21)}$.

No que concerne ao grau de escolaridade detectado, a maioria era não alfabetizada e possuía Ensino Fundamental incompleto (107/36.52\%). A predominância da baixa escolaridade no CAPS também foi observada em outros trabalhos(16,22-24) e pode ser justificada pelo fato de que a maior parte dos usuários do Sistema Único de Saúde apresenta baixa instrução(25). Embora em processo de melhoria, ainda há considerável taxa de pessoas com escolarização deficiente no Brasil, sendo o Nordeste a região com maior índice de analfabetismo e menor média de anos de estudo(26). Outras explicações seriam que pessoas com a saúde mental comprometida apresentam dificuldades de exercer trabalhos produtivos e de explorar seu potencial(2). Além disso, esses indivíduos encontram barreiras em instituições de ensino, sendo, muitas vezes, excluídos do processo de educação e, consequentemente, do mercado de trabalho(2).

Já em relação ao estado civil, a maioria dos usuários (63,14\%) encontrava-se sem companheiro (a) (solteiro/ divorciado/viúvo), sendo esse achado corroborado por outros estudos ${ }^{(15-16,22)}$. Sobre a relação saúde mental e relacionamento amoroso, estudos mostram que o impacto desse tipo de relacionamento dependeria de sua natureza, ou seja, se pautado em maturidade, diálogo, reciprocidade, entre outros aspectos, ele seria benéfico ao indivíduo com saúde mental, pois estimularia sua autonomia e crescimento pessoal. Caso contrário, predisporia ao adoecimento psíquico(27). Por outro lado, o estado civil pode ser consequência das características de certos transtornos mentais. A esquizofrenia, por exemplo, instala-se geralmente em pacientes entre dez e 25 anos e pode cursar com retraimento social, apatia e perda de interesses, que são fatores que podem dificultar a construção de um relacionamento mais estável. Dessa forma, a maioria desses não se casa(28).

A predominância do sexo feminino no CAPS II do município de Paulo Afonso está em consonância com outros trabalhos nesse tipo de serviço(14-18) e pode estar relacionada ao fato de que os homens, em geral, buscam menos os serviços de saúde devido a barreiras culturais e institucionais(29). Somada a isso, revisão sistemática sobre a prevalência dos transtornos mentais na população adulta brasileira mostrou que o sexo masculino é mais acometido por transtornos relacionados ao consumo de substâncias psicoativas, ou seja, seria esperado que houvesse predominância de homens nos Centros de Atenção Psicossocial Álcool e Drogas (CAPS- ad) do município do estudo, e não no CAPS II ${ }^{(19)}$. Estudo realizado nos CAPS - ad de Curitiba/ PR obteve maior prevalência do sexo masculino(30), o que vai ao encontro dessa hipótese.

Em relação aos transtornos prevalentes nas mulheres, estudou verificou que o sexo feminino é mais acometido por transtornos de humor, de ansiedade e somatoformes ${ }^{(19)}$, o que corrobora os achados deste manuscrito, uma vez que os transtornos do humor (F30-F39) e os transtornos neuróticos, relacionados com o estresse e somatoformes (F40-F48), também se mostraram predominantes no sexo feminino $(p=0,00)$ no CAPS II de Paulo Afonso/BA.

A categoria de CID mais prevalente neste estudo, que foi a dos transtornos de esquizofrenia, esquizotípicos e delirantes $(121 / 41,3 \%)$, também predominou em outros levantamentos(17,22,31-32). Dos usuários com esses transtornos, 69 indivíduos $(57,02 \%)$ eram do sexo masculino, o que vai de encontro à epidemiologia 
característica da esquizofrenia, cuja prevalência em ambos os sexos é equivalente(33). Entretanto, esse achado pode ser justificado pelo fato de a esquizofrenia, quando em homens, ter início mais precoce ${ }^{(28)}$, apresentar duração maior e estar associada a um pior

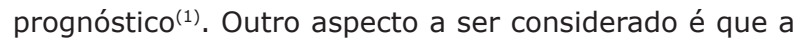
maior prevalência desse grupo do CID no estudo condiz com o serviço prestado pelos CAPS, os quais devem realizar atendimento à população com transtornos mentais graves e persistentes(7). Diferentemente, na atenção básica, os transtornos depressivos e de ansiedade são os mais encontrados ${ }^{(34)}$.

Outro aspecto clínico observado foi a predominância do regime de tratamento não intensivo (210/71,67\%), o qual também foi prevalente em estudo realizado no CAPS do município de Ilhéus/BA ${ }^{(31)}$. Os tipos de atendimento dependem do quadro clínico do indivíduo, ou seja, são definidos a partir de uma análise individualizada da situação de cada usuário. $O$ atendimento intensivo necessita de acompanhamento diário; o semi-intensivo, frequente e o não intensivo, menos frequente ${ }^{(9)}$. No CAPS II do munícipio em estudo, o regime intensivo tem acompanhamento diário, o semi-intensivo, duas vezes na semana e o não intensivo, de 15 em 15 dias ou de dez em dez dias, a depender do caso.

Quanto à forma de entrada no CAPS em estudo, a maioria foi feita por meio de encaminhamentos $(165 / 56,31 \%)$, uma prática comum no Sistema Único de Saúde (SUS), uma vez que tem como princípio organizacional a hierarquização dos serviços ${ }^{(35)}$. Entretanto, alguns estudiosos defendem que esse modelo de sistema de referência e contrarreferência está relacionado a uma fragmentação da assistência, a qual tende a levar a uma desresponsabilização de quem encaminhou(36). Para tal problemática, uma alternativa seria a instalação de um serviço matricial, que "consiste nas ações de supervisão, atendimento compartilhado e capacitação em serviço, realizado por uma equipe de saúde mental para equipes ou profissionais da atenção básica"(37). Isso seria fundamental para a corresponsabilização do cuidado pelas equipes de referência em saúde mental e da atenção básica(37).

A ausência de informações nos prontuários médicos, principalmente sobre ocupação, nível de escolaridade e etnia, foi um ponto limitante da pesquisa. Outra limitação que merece destaque é que variáveis sujeitas a modificações ao longo do tempo - grau de escolaridade, ocupação e estado civil - só constam uma vez no prontuário, ou seja, refletem a situação do indivíduo quando foi admitido no serviço, que não necessariamente condiz com a atual realidade.

Apesar disso, o estudo é de suma importância para a caracterização e análise dos usuários do CAPS II do município de Paulo Afonso/BA e para a fundamentação de atividades terapêuticas no serviço, discussões sobre gestão e sensibilização acerca da importância de se conhecer, registrar e atualizar os aspectos sociodemográficos dos usuários. Ressalta-se que são imprescindíveis mais estudos no CAPS desse município visto que outros aspectos relacionados aos transtornos mentais ainda podem ser pesquisados, como eventos desencadeantes, idade do diagnóstico, taxa de internação e aspectos socioeconômicos.

\section{Conclusão}

A pesquisa permitiu caracterizar os usuários do CAPS II do município de Paulo Afonso/BA, sendo encontrada a maior prevalência de indivíduos com ocupação, do sexo feminino, solteiros, de faixa etária entre 40 e 49 anos, com baixa escolaridade, admitidos por encaminhamento, em atendimento não intensivo e com transtornos do grupo dos transtornos esquizofrênicos, esquizotípicos e delirantes.

Nesse sentido, considerando que os serviços de saúde mental se encontram em processo de aprimoramento, espera-se que este trabalho, pioneiro no CAPS II de Paulo Afonso/BA, seja utilizado como subsídio para o planejamento de intervenções direcionadas a certos grupos e para a elaboração de estratégias de melhorias, além de fomentar novas pesquisas nesse cenário.

\section{Agradecimentos}

Agradecemos a equipe do Centro de Atenção Psicossocial II do município de Paulo Afonso/BA pelo acolhimento durante a coleta de dados e pelo cuidado prestado aos usuários do serviço.

\section{Referências}

1. American Psychiatric Association. Manual diagnóstico e estatístico de transtornos mentais: DSM-5. 5. ed. Porto Alegre: Artmed; 2014. p. 20.

2. Chan M. Mental health and development: targeting people with mental health conditions as a vulnerable group. Geneva: World Health Organization; 2010.

3. World Health Organization. The World health report 2001: Mental health: new understanding, new hope. Geneva: World Health Organization; 2001.

4. Ministério da Saúde (BR). Secretaria de Atenção à Saúde. Departamento de Atenção Básica. Caderno de atenção básica: Saúde Mental. Brasília, DF; 2013.

5. Oliveira AGB de, Alessi NP. Citizenship: instrument and finality of the working process in psychiatric reform. Ciênc Saúde Coletiva. [Internet]. 2005 Jan/Mar; 10(1):191203. [cited Ago 4 2018]. Available from: <http://dx.doi. org/10.1590/S1413-81232005000100026>.

6. Ministério da Saúde (BR). Secretaria de Atenção à Saúde. Departamento de Ações Programáticas 
Estratégicas. Coordenação Geral de Saúde Mental. Reforma psiquiátrica e política de saúde mental no Brasil. Conferência Regional de Reforma dos Serviços de Saúde Mental: 15 anos depois de Caracas. Brasília, DF; 2005.

7. Ministério da Saúde (BR). Portaria no 3.088, de 23 de dezembro de 2011. Republicada em 21 de maio de 2013. Institui a Rede de Atenção Psicossocial para pessoas com sofrimento ou transtorno mental e com necessidades decorrentes do uso de crack, álcool e outras drogas, no âmbito do Sistema Único de Saúde (SUS). Brasília, DF. [Internet]. 2011. [Acesso 5 ago 2018] Disponível em: <http://bvsms.saude.gov.br/bvs/saudelegis/gm/2011/ prt3088_23_12_2011_rep.html>.

8. Ministério da Saúde (BR). Secretaria de Atenção à Saúde. Departamento de Ações Programáticas Estratégicas. Saúde mental no SUS: os centros de atenção psicossocial. Brasília, DF; 2004.

9. Ministério da Saúde (BR). Portaria no 336, de 19 de fevereiro de 2002. Brasília, DF. [Internet]. 2011. [Acesso 3 ago 2018] Disponível em: <http://bvsms.saude.gov.br/ bvs/saudelegis/gm/2002/prt0336_19_02_2002.html>.

10. Instituto Brasileiro de Geografia e Estatística [homepage na internet]. Paulo Afonso. [Acesso 9 ago 2018]. Disponível em: <https://cidades.ibge.gov.br/ brasil/ba/paulo-afonso/panorama $>$.

11. Cadastro Nacional de Estabelecimentos de Saúde (CNES) [homepage na internet]. Estabelecimento de Saúde do Município: Paulo Afonso. [Acesso 9 ago 2018]. Disponível em: <http://cnes2.datasus.gov.br/Lista_Es_ Municipio.asp?VEstado $=29 \&$ VCodMunicipio $=292400 \&$ No meEstado=BAHIA>.

12. Ministério da Saúde (BR). Departamento de Informática do SUS - DATASUS [homepage na internet]. Classificação Internacional de Doenças. Capítulo V Transtornos mentais e comportamentais (F00-F99). [Acesso 9 jul 2018]. Disponível em: <http://www. datasus.gov.br/cid10/V2008/WebHelp/f00_f99.htm>.

13. Altman DG. Practical statistics for medical research. Florida: CRC Press; 1990.

14. Oliveira VF de, Alves JS, Moraes ACS de, Silva JC, Silva CSS da, Nepomuceno FWAB, et al. Clinical characterization of patients with mental disorders assisted in psychosocial care center in São Francisco do Conde - Bahia. Rev Ciênc Méd Biol. [Internet]. 2014;13(2):204-11. [cited Jul 20 2018]. Available from: <http://dx.doi.org/10.9771/cmbio.v13i2.11672>.

15. Soares AC, Trindade L, Rodrigues GCS, Silva FPA da, Sia EF. Clinical-epidemiological analysis of patients with mental disorders in the Brazilian Amazon. Rev Bras Neurol Psiquiatr. [Internet]. 2015; 19(2):96-107. [cited Jul 15 2018]. Available from: <https://rbnp.emnuvens. com.br/rbnp/article/view/55>.

16. Paula CTC. Profile epidemiological of the users of the Psychosocial Care Center in the city of Recife. Cad
Bras Saúde Mental. [Internet]. 2010;2(4-5):94-106. [cited Jul 20 2018]. Available from: <http://incubadora. periodicos.ufsc.br/index.php/cbsm/article/view/1106>. 17. Cruz, LS da, Carmo DC do, Sacramento DMS do, Almeida MSP de, Silveira HF da, Ribeiro HL Junior. Profile of patients with mental disorders assisted in the center for psychosocial care in the city of Candeias, Bahia, Brazil. Rev Bras Ciênc Saúde. [Internet]. 2016;20(2):93-8. [cited Ago 20 2018]. Available from: <https://doi. org/10.4034/RBCS.2016.20.02.01>.

18. Bellettini F, Gomes KM. Profile of attendees of the psychosocial care center and mental health program in the city of Orleans-SC. Cad Bras Saúde Mental. [Internet]. 2013;5(12):161-175. [cited Ago 4 2018]. Available from: <http://incubadora.periodicos.ufsc.br/ index.php/cbsm/article/view/1694/3192>.

19. Santos EG dos, Siqueira MM de. Prevalence of mental disorders in the Brazilian adult population: a systematic review from 1997 to 2009. J Bras Psiquiatr. [Internet]. 2010;59(3):238-46. [cited Ago 4 2018]. Available from: <http://dx.doi. org/10.1590/S0047-20852010000300011>.

20. Zambroni-de-Souza PC. Working with health: work and severe mental disorder. Psicol Estud. [Internet]. 2006;11(1):175-183. [cited Ago 12 2018]. Available from: <http://dx.doi.org/10.1590/ S1413-73722006000100020>.

21. Negrini A, Corbière M, Fortin G, Lecomte T. Psychosocial well-being construct in people with severe mental disorders enrolled in supported employment programs. Commun Ment Health J. [Internet]. 2014;50(8):932-42. [cited July 8 2019]. Available from: <https://doi.org/10.1007/s10597-014-9717-8>.

22. Ballarin MLGS, Miranda IMS de, Carvalho CM de. Psychosocial Care Center in Campinas: a study on the socio-demographic and clinical profiles of its users. Rev Ciênc Méd. [Internet]. 2011; 20(3-4):59-67. [cited Jun 12 2018]. Available from: <https://doi. org/10.24220/2318-0897v20n3/4a581>.

23. Carvalho MDA de, Oliveira HS, Rodrigues LV. Epidemiological profile of users of the municipal Mental Health Care Network of Iguatu, Ceará, Brazil. SMAD Rev Eletron Saúde Mental Álcool e Drogas. [Internet]. 2010; 6(2):337-49. [cited jun 22 2018]. Available from: <https:// doi.org/10.11606/issn.1806-6976.v6i2p337-349>.

24. Mangualde AAS, Botelho CC, Soares MR, Costa JF, Junqueira ACM, Vidal CEL. Epidemiological profile of patients treated in a Center for Psychosocial Care. Mental. [Internet]. 2013; 10(19):235-48. [cited Jun 22 2018]. Available from: <http:// pepsic.bvsalud.org/scielo.php?script=sci_arttext\&pi $d=S 1679-44272012000200006$ > .

25. Silva ZP da, Ribeiro MCSA, Barata RB, Almeida MF de. Socio-demographic profile and utilization patterns of 
the public healthcare system (SUS), 2003- 2008. Ciênc Saúde Coletiva. [Internet]. 2011;16:3807-16. [cited Jun 15 2018]. Available from: <http://dx.doi.org/10.1590/ S1413-81232011001000016>.

26. Instituto Brasileiro de Geografia e Estatística. Pesquisa nacional por amostra de domicílios: síntese de indicadores 2015. Coordenação de Trabalho e Rendimento. Rio de Janeiro: IBGE; 2016.

27. Schlösser A. Interface between mental health and romantic relationship: a look from the positive psychology. Pensando Familias. [Internet]. 2014;18(2):17-33. [cited Jun 17 2018]. Available from: <http://pepsic.bvsalud.org/scielo.php?script=sci_ arttext\&pid=S1679-494X2014000200003>.

28. Fontana AM. Esquizofrenia, Transtornos Esquizotípico e Delirantes. Manual de clínica em psiquiatria. São Paulo: Editora Atheneu; 2006. p. 277-294.

29. Gomes R, Nascimento EF do, Araújo FC de. Why do men use health services less than women? Explanations by men with low versus higher education. Cad Saúde Pública. [Internet]. 2007;23(3):565-74. [cited 12 Jun 2018]. Available from: <http://dx.doi.org/10.1590/ S0102-311X2007000300015>.

30. Ribeiro DR, Carvalho DS de. Drug use of groups in distinct phases of treatment in Psychosocial Care Centers for Alcohol and Drug (CAPS-AD). J Bras Psiquiatr. [Internet]. 2015;64(3):221-2. [cited Jun 12 2018]. Available from: <http://dx.doi. org/10.1590/0047-2085000000082>.

31. Freitas AA, Souza RC de. Clinical and social demographic characterization of Psychosocial Care Centers (CAPS) users. Rev Baiana Saúde Pública. [Internet]. 2010;34(3):530-43. [cited Jun 29 2018]. Available from: <https://doi.org/10.22278/23182660.2010.v34.n3.a53>.

32. Andreoli SB, Ronchetti SSB, Miranda AP de, Bezerra CRM, Magalhães CCPB, Martin D, et al. Utilization of community mental health services in the city of Santos, São Paulo, Brazil. Cad Saúde Pública. [Internet]. 2004;20(3):836-44. [cited Jun 23 2018]. Available from: <http://dx.doi.org/10.1590/ s0102-311X2004000300021>.

33. Sadock BJ, Sadock VA. Compêndio de psiquiatria: ciências do comportamento e psiquiatria clinica. 9. Ed. Porto Alegre: Artmed; 2007.
34. Souza LGS, Menandro MCS, Couto LLM, Schimith PB, Lima RP de. Mental Health in the Family Health Strategy: a review of Brazilian literature. Saúde Soc. [Internet]. 2012;21(4):1022-34. [cited Jun 22 2018]. Available from: <http://dx.doi.org/10.1590/ S0104-12902012000400019>.

35. Ministério da Saúde (BR). Secretaria Nacional de Assistência à Saúde. ABC do SUS: doutrinas e princípios. Brasília, DF; 1990.

36. Zambenedetti G, Perrone CM. Building a process of mental health care network: challenges and potentialities of the Psychiatric Reform. Physis. [Internet]. 2008;18(2):277-93. [cited Jun 17 2018]. Available from: <http://dx.doi.org/10.1590/ S0103-73312008000200005>.

37. Ministério da Saúde (BR). Secretaria de Atenção à Saúde. Departamento de Ações Programáticas Estratégicas. Saúde Mental no SUS: acesso ao tratamento e mudança do modelo de atenção. Relatório de Gestão 2003-2006.: Brasília, DF: Ministério da Saúde; 2007.

\section{Contribuição dos autores}

Concepção e planejamento do estudo: Camila Gomes Barbosa, Paulo Roberto Marinho Meira, Joida Silva Nery. Obtenção dos dados: Camila Gomes Barbosa, Bruno Bezerra Godim. Análise e interpretação dos dados: Camila Gomes Barbosa, Paulo Roberto Marinho Meira, Joida Silva Nery, Bruno Bezerra Godim. Redação do manuscrito: Camila Gomes Barbosa, Paulo Roberto Marinho Meira, Joida Silva Nery, Bruno Bezerra Godim. Revisão crítica do manuscrito: Camila Gomes Barbosa, Paulo Roberto Marinho Meira, Joida Silva Nery, Bruno Bezerra Godim.

Todos os autores aprovaram a versão final do texto.

Conflito de interesse: os autores declaram não haver conflito de interesse. 